\title{
Propuesta metodológica para efectuar un proceso de autoevaluación por conglomerado en carreras universitarias ${ }^{1}$
}

\author{
Methodological Proposal to Conduct a Conglomerate Self-Assessment Process \\ in University Careers ${ }^{2}$
}

\section{Proposta metodológica para realizar um processo de autoavaliação por conglomerado em carreiras universitárias ${ }^{3}$}

María Morera-Castro Universidad Nacional Escuela de Ciencias del Movimiento Humano y Calidad de Vida Heredia, Costa Rica mmore@una.cr https://orcid.org/0000-0003-2218-179X

Christian Azofeifa-Mora
Universidad Nacional
Escuela de Ciencias del Movimiento Humano y Calidad de Vida
Heredia, Costa Rica
cristian.azofeifa.mora@una.cr
(iD) https://orcid.org/0000-0001-8085-9584
Viviana Gómez-Barrantes
Universidad Nacional
Vicerrectoría de Docencia
Heredia, Costa Rica
viviana.gomez.barrantes@una.cr

\footnotetext{
'Este documento pertenece a una serie de artículos producto de las experiencias de los procesos de autoevaluación, acreditación y compromiso de mejoramiento vivenciados por la Comisión de Acreditación de la Escuela de Ciencias del Movimiento Humano y Calidad de Vida del 20132017. Derivados de esta experiencia se publicaron dos artículos adicionales: Calidad y excelencia académica universitaria por conglomerado: Una experiencia de equipo y Propuesta metodológica del análisis de similitudes de carreras de grado para una acreditación por conglomerado.

${ }^{2}$ This document is part of a series of articles that are a product of experiences of self-evaluation, accreditation, and commitment of improvement processes lived by the Commission on Accreditation of the School of Sciences of the Human Movement and Quality of Life of 2013-2017. As a result of this experience, two other papers were published: Academic Quality and Academic Excellence Through Conglomerates: A Team Experience and Methodological Proposal of Similarities Analysis of Degree Courses for Accreditation Through Conglomerates.

${ }^{3}$ Este documento pertence a uma série de artigos, produto das experiências dos processos de autoavaliação, acreditação e compromisso de melhoria experimentados pela Comissão de Acreditação da Escola de Ciências do Movimento Humano e Qualidade de Vida de 2013-2017.Dois artigos adicionais foram publicados como resultado desta experiência : Qualidade acadêmica universitária e excelência por conglomerado: Uma experiência de equipe e Proposta metodológica para a análise de semelhanças de cursos de graduação para acreditação por conglomerado.
} 
Resumen: El objetivo de este artículo fue sistematizar la experiencia metodológica vivida en el proceso de autoevaluación por conglomerado de carreras de grado universitarias que se encuentran en diferentes etapas de evaluación y que cuentan con dos unidades ejecutoras. Esta propuesta incluye las fases y etapas que exponen los detalles metodológicos, entre ellos: diseño de plan de acciones, reuniones permanentes colectivas para la sistematización y toma de decisiones, diseño de herramientas para estandarizar el proceso de acreditación por conglomerado. Como resultado de la aplicación metodológica se concluyen como estrategia exitosa la forma de trabajo establecida por la comisión, la cual permitió una cohesión de grupo, sentido de pertenencia y apropiación del proceso. Este artículo puede ser utilizado como una guía para orientar las actividades académicas de autoevaluación y mejoramiento, en carreras en aras de ser acreditas, que poseen un alto grado de afinidad entre ellas, ya sea desde su objeto de estudio o proceso educativo.

Palabras claves: Calidad educativa; educación superior; acreditación; control de calidad; gestión de calidad total.

Abstract: The purpose of this article was to systematize the methodological experience lived in the conglomerate process of self-evaluation of university graduate careers, which are in different stages of evaluation and have two executing units. This proposal includes the phases and stages that have all the methodological details, including design of an action plan, permanent collective meetings for the systematization and decision-making, and design of tools to standardize the accreditation process by conglomerate mainly. As a result of this methodological application, the form of work established by the commission is concluded as a successful strategy; it allowed a group cohesion, sense of belonging, and appropriation of the process. This article can be used as a document to guide the academic activities of self-evaluation and improvement in careers that have a high degree of affinity between them, either from their object of study or educational process, in order to be accredited.

Keywords: Educational quality; higher education; accreditation; quality control; total quality management. 


\begin{abstract}
Resumo: O objetivo deste artigo foi sistematizar a experiência metodológica vivenciada no processo de autoavaliação por conglomerados de cursos de graduação que estão em diferentes estágios de avaliação e que possuem duas unidades executoras. Essa proposta inclui as fases e etapas que expõem os detalhes metodológicos, entre elas: desenho do plano de ação, reuniões coletivas permanentes para sistematização e tomada de decisão, desenho de ferramentas para padronizar o processo de acreditação por conglomerado. Como resultado da aplicação metodológica, conclui-se como estratégia bem-sucedida a forma de trabalho estabelecida pela comissão, o que permitiu uma coesão grupal, sentimento de pertença e apropriação do processo. Este artigo pode ser utilizado como um guia para orientar as atividades acadêmicas de autoavaliação e aprimoramento, em carreiras passadas pelo processo de acreditação, que tenham um alto grau de afinidade entre elas, seja a partir de seu objeto de estudo ou processo educacional.
\end{abstract}

Palavras-chave: Qualidade educacional; educação superior; acreditação; controle de qualidade; gestão da qualidade total.

\title{
Introducción
}

Debido a la elevada demanda de la sociedad, el sector productivo, el sector servicios y el Estado mismo, se ha requerido implementar controles estrictos de calidad en los procesos de enseñanza de la educación superior (Villarruel, 2010). Para que la educación universitaria dé respuesta a la forma en que el aprendizaje se concibe actualmente, sea innovadora y creadora de conocimiento, así como generadora de vínculos entre la sociedad y el mundo laboral, es necesario el análisis de sus objetivos de manera permanente, conscientes y a largo plazo (Acosta y Acosta, 2016; Hernández, 2014).

De ese modo, la autoevaluación de la educación, y en este caso de la educación superior, es una forma de identificar fortalezas y debilidades en busca de una mejor calidad en un programa dentro del sector educación (Marciniak y Gairín, 2017). Para Gairín, Díaz-Vicario, Rosales y Sentinella (2014), estos procesos se justifican por la responsabilidad que tienen las personas profesionales sobre la mejora de la educación y de los programas que desarrollan. A su vez, señalan lo imprescindible que es la evaluación para la revisión, el monitoreo, la identificación y el mejoramiento de aspectos curriculares, organizativos, contextuales y de gestión que permitan asumir compromisos que optimicen y fortalezcan la calidad de la formación profesional contextualizada en las demandas y necesidades de la sociedad.

Por su parte, López-Rodríguez, González-Maura y Valdivia-Díaz (2016) hacen notar que los modelos de evaluación de la calidad para la mejora continua son importantes y novedosos en tanto propicien la participación y compromiso de diferentes personas actoras, como el personal académico, autoridades u otras. Además, mencionan que estos procesos participativos, de rigor científico y colaborativos, generan una cultura de autoevaluación con impacto en la formación, la docencia y los procesos administrativos de una institución. 
doi: http://dx.doi.org/10.15359/ree.23-3.13

URL: http://www.una.ac.cr/educare

CORREO: educare@una.cr

Sin embargo, es claro que los procesos de autoevaluación son complejos; en estos se deben abordar las normativas, reglamentos, dinámicas de gestión de la calidad y contemplar problemáticas y necesidades específicas que no siempre son coincidentes entre sí (Villarruel, 2010). En la educación superior estos procesos de autoevaluación de carreras o programas universitarios, ciertamente, poseen un grado de complejidad, debido a la naturaleza particular de cada carrera y se puede incrementar aún más si interactúan con más de una unidad académica, más de una carrera o más de una universidad (Charpentier-Esquivel, HernándezSalazar, Fedorov-Fedorov y Rojas-Sandí, 2016).

En Costa Rica, el Sistema Nacional de Acreditación de la Educación Superior (SINAES), con el fin de diversificar su quehacer y brindar opciones más coherentes con el funcionamiento de las instituciones de educación superior, creó un modelo de proceso de autoevaluación por conglomerado, que permite el análisis y la evaluación de aquellas carreras con cierto grado de afinidad en sus características de constitución y funcionamiento y así minimizar el costo y tiempo requerido para autoevaluar (SINAES, 2013b). Para optar por dicha acreditación es necesario realizar una primera revisión de las carreras a autoevaluar, a fin de determinar su grado de afinidad y, posteriormente, realizar un proceso de autoevaluación en donde se identifican características, fortalezas y procesos de mejora en cada carrera durante los últimos cuatro años. Dicho proceso se sustenta en el seguimiento de una serie de estándares y criterios emanados por este ente acreditador que permite la redacción y conformación del informe de autoevaluación, documento presentado ante el ente evaluador para determinar el grado de calidad de las carreras y definir si pueden obtener el certificado de calidad.

Es aquí donde la Escuela de Ciencias del Movimiento Humano y Calidad de Vida (CIEMHCAVI) de la Facultad de Ciencias de la Salud, de la Universidad Nacional (UNA), tomó la decisión de acogerse a esta posibilidad para acreditar las carreras de grado que ofrece. Esta decisión la toma la Escuela basada en la experiencia que obtuvo durante la realización de un primer proceso de autoevaluación y ejecutó un Compromiso de Mejoramiento (CM) por cuatro años que permitió la obtención de la acreditación del Bachillerato en la Enseñanza de la Educación Física, Deporte y Recreación (BEEFDR) ante el Sistema Nacional de Acreditación de la Educación Superior. Esta es una carrera compartida con otra escuela de la Universidad Nacional denominada División de Educología, perteneciente al Centro de Investigación y Docencia en Educación, que de manera conjunta y con un modelo de formación simultáneo, forman docentes en el área de la Educación Física, Deporte y Recreación.

Esta experiencia propició en la CIEMHCAVI procesos de mejoramiento que impactaron toda la oferta académica que incluye bachilleratos, licenciaturas, maestrías y doctorado, por lo que para la siguiente autoevaluación, que aspiraba a la reacreditación del BEEFDR, se determinó hacer un proceso de acreditación por conglomerado que integrara también el Bachillerato en Promoción de la Salud Física (BPSF), de manera que la autoevaluación permitiera demostrar la calidad de ambas carreras. María Morera-Castro, Christian Azofeifa-Mora, Viviana Gómez-Barrantes, Ana Azofeifa-Lizano y Daniel Rojas-Valverde

Los artículos de la Revista Electrónica Educare del Centro de Investigación y Docencia en Educación de la Universidad Nacional, Costa Rica, se comparten bajo términos de la Licencia Creative Commons: Reconocimiento, No Comercial, Sin Obra Derivada 3.0 Costa Rica. Las autorizaciones adicionales a las aquí delimitadas se pueden obtener en el correo: educare@una.cr 
Por lo tanto, el propósito de este artículo es describir la metodología empleada en el proceso de autoevaluación para la acreditación por conglomerado de dos carreras de grado, que se encuentran en diferentes etapas de valoración y que cuentan con dos unidades ejecutoras.

\section{Metodología que integra las estrategias para un proceso de autoevaluación por conglomerado}

A continuación, se presenta por fases la estrategia metodológica que la Comisión de Conglomerado de la Escuela de Ciencias del Movimiento Humano y Calidad de Vida utilizó en las diferentes etapas del proceso de autoevaluación para la acreditación por conglomerado de dos carreras de grado, en su efecto BEEFDR y BPSF. Este proceso fue liderado por la CIEMHCAVI, en conjunto con la División de Educología, instancia universitaria que se encarga de desarrollar el componente pedagógico en las carreras de formación en la enseñanza, como es el caso del BEEFDR.

\section{Primera fase: Creación de una comisión interdisciplinar}

Como parte del proceso de organización se creó una comisión de acreditación por conglomerado, la cual se denominó Comisión de Conglomerado CIEMHCAVI (CCC). Esta estuvo conformada por:

1. Las personas que ocupaban el puesto de subdirección de la Escuela de Ciencias del Movimiento Humano y Calidad de Vida y la División de Educología.

2. Una persona con el puesto de coordinadora de la comisión.

3. Tres personas académicas con conocimientos en la ejecución de las dos carreras. Dos de las cuales pertenecía a la CIEMHCAVI y una a la DE.

4. Una persona asesora del Proceso de Seguimiento y Evaluación de Carreras de la Vicerrectoría de Docencia, de la UNA.

5. Una persona representante estudiantil, la cual fue contratada bajo la figura de estudiante asistente.

6. Una persona designada del personal administrativo de la CIEMHCAVI.

\section{Segunda fase: Cumplimiento de normativa institucional}

Según la normativa institucional de la Universidad Nacional, se formuló una actividad académica denominada "Gestión de la Calidad CIEMHCAVI", en la cual se definen propósitos específicos que sirven como medio de coordinación y articulación entre las diferentes 
doi: http://dx.doi.org/10.15359/ree.23-3.13

URL: http://www.una.ac.cr/educare

CORREO: educare@una.cr

autoridades universitarias y los diferentes sujetos actores de la CIEMHCAVI que permitan apoyar y contribuir en la implementación de parámetros de referencia, estrategias de gestión, objetivos, actividades, entre otros, que fortalezcan los procesos de autoevaluación, acreditación, reacreditación y mejoramiento de la calidad de las carreras ofertadas por la Escuela.

\section{Tercera fase: Modelo de trabajo establecido por la CCC}

La CCC estableció como política interna reunirse, de forma semanal y permanente, en pleno, con la intensión de que, en forma conjunta, se coordinaran las acciones necesarias que permitieran culminar cada etapa propuesta en el plan de acción.

Durante estos espacios, si bien es cierto se delegaban funciones específicas a cada una de las personas miembros de la CCC para su cumplimiento entre la semana, el principal objetivo fue la creación en conjunto de toda la documentación requerida en el proceso de autoevaluación, entre ellos se pueden citar: elaboración de encuestas, informes de análisis de resultados de las encuestas, elaboración de informe de autoevaluación y $\mathrm{CM}$, búsqueda y determinación de evidencias, revisión de la documentación por el ente acreditador y cualquir otra documentación, evidencia o acción que se necesitara.

La dinámica que prevaleció en estas reuniones fue la participación proactiva de la totalidad de miembros en la toma de decisiones, gracias a un liderazgo democrático y a la determinación de roles de trabajo para sus integrantes, de acuerdo con las fortalezas de cada uno de sus miembros.

Lo anterior permitió la apropiación conjunta, la participación activa y propositiva de cada una de las personas de la CCC y una plena consciencia de su rol en cada momento. Asimismo, se generaron procesos de realimentación permanentes con base en los distintos puntos de vista de cada participante, lo cual propició un trabajo más armónico e integral con sensibilización, contextualización y concientización más completa en cada integrante; esto permitió un producto realizado de una forma más ágil y eficiente.

La sistematización de esta experiencia vivida se encuentra disponible en Morera-Castro, Azofeifa-Lizano, Gómez-Barrantes, Rojas-Valverde y Azofeifa-Mora (2017).

\section{Cuarta fase: Plan de acción establecido por la CCC}

Se estableció un plan de acción general para la ejecución del proceso de autoevaluación por conglomerado. Este plan incluyó un cronograma de trabajo que contemplaba: los recursos humanos y materiales disponibles, necesidades del momento a nivel de unidades académicas y los plazos de cumplimiento internos, institucionales y del ente acreditador. Asímismo, este plan se dividió en dos etapas macro: 


\section{Etapa I}

El análisis del grado de afinidad de las carreras involucradas. Que a su vez incluyó:

a. Análisis del grado de afinidad de las carreras.

b. Elaboración del informe correspondiente.

c. Solicitud, ante el ente acreditador correspondiente, del aval para optar por una acreditación por conglomerado.

La sistematización del análisis de similitudes se encuentra disponible en Morera-Castro, Gómez-Barrantes, Azofeifa-Lizano, Rojas-Valverde y Azofeifa-Mora (2018). Como una síntesis de este artículo se estableció una metodología específica que permitiera analizar todas las evidencias en común entre ambas carreras, para determinar si se contaba con el porcentaje mínimo requerido por el ente acreditador, en cada una de las dimensiones establecidas en función del Manual de acreditación que determina los criterios y estándares de evaluación.

Con el análisis se demostró el alto grado de afinidad entre las carreras, lo que le posibilitó a la CIEMHCAVI iniciar con el proceso de obtención de los avales ante los órganos colegiados respectivos tanto de los Consejos y las Asambleas de Escuela, así como de las instancias universitarias correspondientes: Vicerrectoría de Docencia y Rectoría, que otorgaran la

aprobación para que se iniciara con la acreditación por conglomerado. Finalmente, se logró la aprobación del ente acreditador para optar por la acreditación por conglomerado.

\section{Etapa II}

El proceso de autoevaluación por conglomerado de las carreras de grado, implicó:

- Sensibilización a las diferentes poblaciones involucradas sobre el proceso de autoevaluación, a su haber, estudiantes de primer ingreso, estudiantes regulares de las carreras, personal académico, personal administrativo, autoridades universitarias de cada Escuela, personas graduadas y entes empleadores

- Recopilación, clasificación y creación de un registro de evidencias que permitieron sustentar el Informe de Autoevaluación por Conglomerado (IA).

- Elaboración, aplicación y análisis de resultados de encuestas de opinión a los sujetos actores del proceso, tomando como base los instrumentos macro creados por el respectivo departamento de la Universidad Nacional.

- Socialización de los resultados de las encuestas a los diferentes grupos participantes.

- Elaboración del Informe final de autoevaluación por conglomerado y CM del BEEFDR y BPSF.

- Entrega del IA ante el ente acreditador. 
doi: http://dx.doi.org/10.15359/ree.23-3.13

URL: http://www.una.ac.cr/educare

CORREO: educare@una.cr

\section{Resultados, análisis y discusión de la etapa II del plan de acción de la CCC}

\section{Sensibilización a las diferentes poblaciones involucradas}

Se coordinó con la dirección de la Escuela para establecer un cronograma de visitas a los diferentes grupos de estudiantes de ambas carreras, con el objetivo de efectuar una inducción a los procesos de autoevaluación y acreditación por conglomerado, el rol de importancia que cada población adquiere y la necesidad de una participación activa por parte del estudiantado. Para facilitar la transmisión el mensaje se crearon presentaciones multimedia, pizarras informativas y publicaciones en redes sociales. Estas mismas estrategias de sensibilización se utilizaron para el personal docente y administrativo.

En el caso de entes empleadores y personas graduadas se actualizaron las bases de datos, seguido de llamadas telefónicas, envío de correos electrónicos y publicaciones en redes sociales, informándoles e invitándoles a ser personas activas de la autoevaluación.

\section{Recopilación de evidencias}

La recopilación de las evidencias fue realizada por la CCC en colaboración con todas las personas que conforman las escuelas o los diferentes departamentos de la universidad.

Las evidencias las constituyeron todas aquellas políticas, reglamentos, normativas, procedimientos, sistemas de información digitales, sistemas de control entre otras que respaldan la gestión institucional. Además, de todas aquellas acciones, directrices, lineamientos, actividades, procedimientos internos, como: hojas de asistencia, actas de escuelas, graduaciones, equiparaciones, fotos, programas de cursos, currículos vitae, informes de proyectos, programas y actividades académicas, capacitaciones, publicaciones y otros elementos asociados con el quehacer de las Unidades Académicas.

Para la búsqueda de esta información la CCC utilizaron diversas estrategias:

1. Se identificaron y analizaron todas las evidencias solicitadas por el ente acreditador.

2. De las evidencias que se poseían producto del proceso de autoevaluación y CM de BEEFDR 2011-2013, se procedió a verificarlas, actualizarlas, digitalizarlas y trasladarlas a las carpetas correspondientes.

3. De las evidencias que se encontraban de manera física, se procedió a digitalizarlas y a incluirlas en las carpetas correspondientes.

4. Se elaboró una lista de las evidencias faltantes por componente y criterio. Una vez localizadas se digitalizaron y acomodaron en las carpetas correspondientes. 
5. Se realizó una búsqueda en línea de los documentos institucionales en los diferentes sitios web de la CIEMHCAVI, División de Educología y UNA. Se copió el link y se colocó según correspondieran en el Informe final de autoevaluación por conglomerado (IA).

6. Se efectuó una recopilación digital por medio de fotografías de aquellas acciones o actividades que no tuvieran un respaldo documental, por ejemplo: giras de cursos, edificaciones nuevas o remodelaciones, rotulación horizontal y vertical, entre otros.

7. Se efectuó una recopilación digital de las evidencias fotográfícas ya existentes producto de las acciones y el quehacer de los proyectos, programas o actividades académicas.

Como resultado se almacenaron dentro de una carpeta general denominada"información general", toda la información concerniente a políticas y normativas institucionales, cuestionarios de opinión y documentos de citación continua en el IA. El restante de las evidencias fue ordenado por carpetas según la dimensión, el componente, el criterio y la evidencia correspondiente establecidos por el ente acreditador.

Cabe resaltar que todo documento utilizado como sustentación de la evidencia requerida se manejó de forma digitalizada o a través de localizador de recursos uniformes, más conocido por la sigla URL, para permitir acceder a la información de manera expedita. Asimismo, toda la información recopilada se renombró siguiendo la nomenclatura establecida por la CCC.

\section{Encuestas de opinión a los diferentes sujetos actores}

Se elaboraron 7 encuestas de opinión con preguntas abiertas y cerradas. Estas se construyeron en función de las personas actoras: personal académico, personal administrativo, personas graduadas, entes empleadores, estudiantes regulares y estudiantes de primer ingreso, para dar respuesta a criterios solicitados y según las necesidades de información de las carreras.

Estas encuestas fueron elaboradas en las reuniones de la CCC con base en el Manual de Acreditación Oficial de Carreras de Grado del Sistema Nacional de Acreditación de la Educación Superior (SINAES, 2009), la Guía para la Autoevaluación de Carreras con fines de acreditación y reacreditación oficial (SINAES, 2011), la Guía para Autoevaluación de carreras por conglomerados (SINAES, 2013a) y las encuestas macro elaboradas por la Universidad Nacional.

Para la confección de los items de cada encuesta, se procedió a analizar las evidencias requeridas por el ente acreditador, y una vez contextualizada y contrastada con las encuestas base de la UNA, se procedió a ajustar, modificar o en caso de ser necesario redactar la pregunta nuevamente. Una vez elaboradas cada una de ellas se revisaron las escalas de opinión propuestas, con el objetivo de garantizar la coherencia entre lo que se solicitaba y la posible respuesta. 
doi: http://dx.doi.org/10.15359/ree.23-3.13

URL: http://www.una.ac.cr/educare

CORREO: educare@una.cr

En el Apéndice A se detallan las secciones que incluye cada encuesta, así como el total de preguntas por sección y el tipo. Esto, con el fin de que las personas interesadas puedan visualizar la distribución temática por grupo poblacional.

La aplicación de las encuestas antes mencionadas se realizó por medio de la plataforma en línea LimeSurvey. Primeramente, se creó una plantilla representativa al proceso donde se incluyeron los logotipos e imagotipos institucionales y de unidades académicas. El siguiente paso consistió en la programación en la plataforma en línea de cada uno de los cuestionarios que habían sido analizados y creados previamente, así como el introducir las bases de datos de las personas participantes. Es necesario clarificar que la plataforma mantiene criterios de confidencialidad permanentes.

Como parte del control de calidad que garantizara el adecuado funcionamiento de cada una de las encuestas diseñadas, se efectuó un riguroso procedimiento de revisión y corrección por parte de integrantes de la CCC y profesionales que colaboraron en este proceso. Este control de calidad se llevó a cabo de la siguiente manera: a) la programación de cada cuestionario mediante la plataforma de LimeSurvey, b) una revisión de su contestación dentro del modo de prueba de la plataforma, c) la presentación de cada cuestionario en las reuniones de la CCC donde se efectuó la simulación de este y se creó un registro con las recomendaciones, sugerencias, cambios y errores por encuesta, según la necesidad, d) estudiante asistente inmerso dentro del proceso de acreditación, como prueba piloto contestó cada cuestionario según su experiencia; en caso de detectar alguna anomalía, incoherencia o que le surgiera duda, debía anotarlo para luego efectuar un reporte con las correcciones necesarias relacionadas con el formato o programación que necesitaran corrección ye) solucionados dichos errores, el cuestionario estaba listo para ser enviado.

Seguidamente, se confeccionaron y digitalizaron las bases de datos mediante el software Microsoft Excel con la información de las personas actoras (personal docente, administrativo, entes empleadores, estudiantado graduado). En el caso particular del estudiantado, se elaboraron dos bases de datos diferentes: estudiantes de primer ingreso y estudiantes regulares, este último grupo incluye al estudiantado que matriculó la mayoría de los cursos entre los niveles II, III y IV.

Paralelamente, se procedió a la importación de cada base de datos a la plataforma de LimeSurvey según el cuestionario correspondiente y se verificó que los datos fuesen importados de forma correcta. Finalizado de forma exitosa este procedimiento, se ejecutó el envío de los cuestionarios a las diferentes poblaciones

Como resultados de la participación de la población involucrada en el proceso, se utilizó una muestra por conveniencia debido a que el objetivo principal era abarcar la mayor cantidad de población posible en cada grupo muestral. Se contó, por tanto, con la participación de un total de 472 personas, las cuales se dividen en: 203 estudiantes regulares de ambas carreras, 63 estudiantes de primer ingreso de ambas carreras, 85 personas graduadas del BEEFDR y 30 personas graduadas del BPSF, 33 del personal académico de la CIEMHCAVI-DE que imparten 
lecciones en alguno de los bachilleratos, 26 personas administrativas tanto de la CIEMCAVI como de la DE y 32 sujetos empleadores.

El detalle muestral de cada encuesta se indica a continuación:

1. Del estudiantado regular de ambas carreras, se invitó a participar a un total de 206 estudiantes, y respondió el $98.56 \%(n=203)$.

2. El total del estudiantado de primer ingreso de ambas carreras a quienes se les envió el cuestionario fue 63 y lo completaron el 100\%.

3. Del personal académico se le aplicó a un total de 33 docentes, 28 de la CIEMHCAVI y 5 de la DE. De esta población se obtuvo un $100 \%$ de participación. Cabe señalar que este cuestionario fue aplicado a todas las personas funcionarias activas de la CIEMHCAVI en el último período de los cuatro años. En el caso de la División de Educología, se aplicó a la totalidad del profesorado activo que imparte lecciones del componente pedagógico de la carrera de BEEFDR. Es importante tomar en cuenta que el personal docente de la CIEMHCAVI imparte lecciones en ambas carreras.

4. Del personal administrativo se tuvo participación de un total de 26 personas de ambas unidades académicas, 24 de CIEMHCAVI y 2 de la DE, logrando un 100\% de la muestra. El personal administrativo de la CIEMHCAVI da apoyo a ambas carreras. Y en el caso del personal de la DE da apoyo al componente pedagógico del BEEFDR. Se contempló a todas aquellas personas que estuvieron colaborando activamente los últimos cuatro años.

5. En el caso de las personas graduadas del BEEFDR se contó con un total de 130 personas meta, de las cuales $65.38 \%(n=85)$ respondió el instrumento y de un total de 70 personas graduadas en el BPSF, se obtuvo respuesta del $42.86 \%(n=30)$. Se invitó a participar a personas graduadas de ambas carreras de los últimos cuatro años.

6. De un total de 40 personas empleadoras, se logró una participación del $80 \%(n=32)$. Para esta muestra en particular se aplicó el muestreo por clúster, con participación representativa de las diferentes instituciones empleadoras de ambas carreras, como por ejemplo: federaciones y asociaciones deportivas, comités cantonales de deporte y recreación, centros de educación primaria y secundaria, públicas y privadas, direcciones regionales del Ministerio de Educación Pública, centros de atención en salud, Comité Olímpico de Costa Rica, centros de acondicionamiento físico, Caja Costarricense del Seguro Social, Instituto Costarricense del Deporte y la Recreación, entre otras empresas e instituciones relacionadas con el área de movimiento humano.

Aplicados los cuestionarios, se procedió a descargar los datos almacenados en la plataforma LimeSurvey. Una vez realizado el proceso anterior, la CCC procedió a la tabulación de la información cuantitativa que fue organizada en tablas de distribución de frecuencias y porcentajes; la información cualitativa correspondiente a las preguntas abiertas de cada 
doi: http://dx.doi.org/10.15359/ree.23-3.13

URL: http://www.una.ac.cr/educare

CORREO: educare@una.cr

cuestionario se organizó por categorías por medio de tablas comparativas, que en conjunto permitieron llevar a cabo un análisis y valoración por dimensión, componente y criterio.

El análisis y tabulación de la información descrita anteriormente dio origen a una serie de informes donde se presentan, de manera detallada, los resultados de cada uno de los cuestionarios aplicados. Estos resultados sirvieron de insumo para la confección del Informe de autoevaluación por conglomerado, específicamente para las evidencias donde se solicita la opinión de las personas actoras.

\section{Socialización a los diferentes actores de los resultados de las encuestas}

Una vez que la CCC finalizó los informes se presentan los resultados de cada una de las encuestas. Como resultados se efectuaron un total de tres talleres con estudiantes, personal académico y administrativo, para dar a conocer los resultados que se consideraron sobresalientes tanto en las fortalezas como en las debilidades encontradas por grupo poblacional. Esto le permitió a la CCC no solo sensibilizar y socializar los resultados, sino también realizar en conjunto con cada población un análisis de las opiniones emitidas, propiciando un enriquecimiento sobre los juicios valorativos a partir de nueva información cualitativa.

\section{Elaboración del informe final del proceso de autoevaluación por conglomerado y compromiso de mejoramiento}

La elaboración del Informe Final de Autoevaluación por Conglomerado integra una versión preliminar del CM según las debilidades encontradas en el proceso de autoevaluación antes descrito. Este documento se elaboró tomando como base los requerimientos que el ente acreditador establece en el Manual de Acreditación Oficial de Carreras de Grado Superior, la Guía para la Autoevaluación de Carreras con fines de Acreditación y Reacreditación Oficial, así como la Guía de Autoevaluación por Conglomerados. Es importante que, para el proceso de redacción, se tomó la decisión de que la comisión en pleno participara. Ello permitió crear una triangulación entre la elaboración del IA, lo solicitado por el ente acreditador y las evidencias.

Debido a la particularidad que tenía este proceso, una carrera acreditada versus una carrera por acreditarse, y donde una de estas carreras es compartida por dos UA, se debieron realizar ajustes y modificaciones al formato del IA, que permitieran dar esa flexibilidad necesaria sin perder la claridad y transparencia de la situación de cada carrera y la atención a los requerimientos del ente acreditador. Este informe fue estructurado de tal forma que conservara el orden según cada dimensión, criterio y evidencia requerida.

Para cada criterio se realizó una descripción y fundamentación de políticas, normativas, lineamientos, reglamentos, procedimientos, el modelo de gestión y datos estadísticos, tanto institucional como de las unidades académicas, que permitieron respaldar el grado de cumplimiento de ambas carreras. Cada insumo fue amparado con las evidencias

12 María Morera-Castro, Christian Azofeifa-Mora, Viviana Gómez-Barrantes, Ana Azofeifa-Lizano y Daniel Rojas-Valverde

Los artículos de la Revista Electrónica Educare del Centro de Investigación y Docencia en Educación de la Universidad Nacional, Costa Rica, se comparten bajo términos de la Licencia Creative Commons: Reconocimiento, No Comercial, Sin Obra Derivada 3.0 Costa Rica. Las autorizaciones adicionales a las aquí delimitadas se pueden obtener en el correo: educare@una.cr 
correspondientes que le dieron objetividad y validez a lo descrito. Seguido de los resultados de las encuestas de opinión de los diferentes sujetos actores involucrados en el desarrollo de las carreras y las referencias a los informes de resultados correspondientes y en caso de ser requerido la evidencia correspondiente al estándar solicitado.

Seguidamente, se efectuó una valoración del criterio en donde se sintetiza las fortalezas y las debilidades presentadas por ambas carreras de lo estipulado en el criterio, así como si el criterio y estándar se cumple, no se cumple o se cumple parcialmente.

Debido a que el BEEFDR se encontraba en un proceso de reacreditación, el ente acreditador solicitaba un análisis comparativo del proceso de autoevaluación pasado y el actual. Este quizás fue el punto que mayor complejidad presentó, ya que se tendía a confundir con los resultados del proceso de autoevaluación por conglomerado. Por lo que la CCC tomó la decisión de crear un apartado en cada criterio que permitiera efectuar una valoración comparativa de las mejoras efectuadas en el BEEFDR, lo cual permitió evidenciar la permanencia de aspectos positivos o bien las mejoras logradas gracias al proceso de acreditación anterior.

A manera de síntesis por dimensión se realizó una tabla resumen de las fortalezas y debilidades del proceso de autoevaluación por conglomerado, esto no incluyó las mejoras del proceso del BEEFDR, excepto que se comprobara una debilidad existente o surgiera una nueva debilidad.

Finalizado cada componente se presentó una síntesis valorativa del componente, así como una síntesis valorativa por dimensión y una valoración integral de las carreras del conglomerado, todos estos solicitados por el ente acreditador. Al mismo tiempo el capítulo final del IA incluye la primera propuesta del CM según las debilidades encontradas en el proceso de autoevaluación por conglomerado y lo evidenciado en el informe.

Es importante señalar que para la entrega del IA al ente acreditador se debió contar con los avales de unidades académicas correspondientes, así como de Rectoría de la Universidad.

\section{Conclusiones}

Luego de vivenciar un proceso de autoevaluación por conglomerado, se concluye que para una exitosa acreditación por conglomerado es necesario:

a. La determinación de sesiones de trabajo recurrentes, lo que implica el establecimiento previo de un plan de acción congruente con las necesidades, recursos, plazos y deberes, así como el involucramiento activo de las personas que conforman la comisión.

b. La participación activa de todos los sujetos actores de las unidades académicas para garantizar el éxito del proceso, la vinculación y la sensibilización permanente de todas las poblaciones permitió una buena cuota de cumplimiento en las metas trazadas para el logro de la certificación de calidad. Es así como el aporte estudiantil se vuelve indispensable, pues refieren al sector que recibe y vivencia la ejecución de las carreras. 
doi: http://dx.doi.org/10.15359/ree.23-3.13

URL: http://www.una.ac.cr/educare

CORREO: educare@una.cr

c. El desarrollo de una metodología propia que responda al contexto y las poblaciones con las que se desea trabajar, de manera que se desarrollen estrategias asertivas que aseguren procesos de autoevaluación transparentes, participativos y exitosos.

d. El orden y la sistematización constantes son aspectos relevantes para la organización y ubicación de la información de cada evidencia, así como para que cada integrante de la comisión genere un conocimiento e involucramiento total del proceso y pueda en cualquier momento asumir responsabilidades sin depender de una sola persona; así se reduce la incertidumbre y la angustia.

e. En el seno de la comisión se debe promover la cohesión de grupo, sentido de pertenencia, espacios de diálogo y apropiación de los requerimientos del proceso.

Se recomienda que la autoevaluación debe estar plagada de voluntad, compromiso, rigurosidad y veracidad, por parte de las personas actoras, la comisión, direcciones de las unidades académicas y autoridades institucionales. A su vez, es fundamental tener conocimiento de los documentos con los cuales trabaja la agencia acreditadora, pero aún más el lograr traducirlos a un lenguaje institucional que permita adecuarlos a las particularidades de cada carrera.

Además, es importante tomar en cuenta, que en las carreras donde existan dos unidades ejecutoras se debe, por tanto, respetar las particularidades del quehacer de cada unidad; sin embargo, se requiere una comunicación y coordinación permanente que garantice que las fortalezas de una unidad puedan permear en la otra o viceversa, es decir, que los aspectos de mejora permitan entrelazar acciones que nutran la gestión de calidad en ambas escuelas.

\section{Referencias}

Acosta, B. y Acosta M. (2016). Modelos de evaluación para la acreditación de carreras. Análisis de su composición y una respuesta para las carreras de Ecuador. Revista Mexicana de Investigación Educativa, 21(71), 1249-1274. Recuperado de http://www.scielo.org.mx/pdf/ rmie/v21n71/1405-6666-rmie-21-71-01249.pdf

Charpentier-Esquivel, C., Hernández-Salazar, C., Fedorov-Fedorov, A. y Rojas-Sandí, M. (2016). Una experiencia interuniversitaria y multidisciplinaria de autoevaluación para la acreditación: Doctorado en Ciencias Naturales para el Desarrollo. Revista Calidad en la Educación Superior, 7(2), 1-24. doi: http://dx.doi.org/10.22458/caes.v7i2.1594

Gairín, J., Díaz-Vicario, A., Rosales, M. y Sentinella, X. (2014). La autoevaluación para la mejora de la seguridad integral en centro educativos. EDUCAR, 50(2), 363-381. doi: https://doi. org/10.5565/rev/educar.144

Hernández, F. (2014). Evaluación y acreditación del profesorado, programas e instituciones educativas. Revista Electrónica Interuniversitaria de Formación del Profesorado, 17(1), 15-32. doi: http://dx.doi.org/10.6018/reifop.17.1.198821 
López-Rodríguez, A., González-Maura, V. L. y Valdivia-Díaz, J. E. (2016). Evaluación interna de la calidad en la universidad de Atacama. Hacia un modelo permanente y participativo para la mejora continua. Revista Calidad en la Educación Superior, 7(2), 180-202. doi: http:// dx.doi.org/10.22458/caes.v7i2.1606

Marciniak, R. y Gairín, J. (2017). Un modelo para la autoevaluación de la calidad de programas de educación universitaria virtual. RED. Revista de Educación a Distancia, 54(2), 1-30. doi: https://doi.org/10.6018/red/54/2

Morera-Castro, M., Azofeifa-Lizano, A., Gómez-Barrantes, V., Rojas-Valverde, D. y AzofeifaMora, C. (2017). Calidad y excelencia académica universitaria por conglomerado: Una experiencia de equipo. Revista Calidad en la Educación Superior, 8(2), 283-308. doi: http:// dx.doi.org/10.22458/caes.v8i2.1901

Morera-Castro, M., Gómez-Barrantes, V., Azofeifa-Lizano, A., Rojas-Valverde, D. y Azofeifa-Mora, C. (2018). Propuesta metodológica para el análisis de similitudes para una acreditación por conglomerado de carreras de grado en Costa Rica. Revista Calidad en la Educación Superior, 9(1), 298-321. doi: http://dx.doi.org/10.22458/caes.v9i1.1941

Sistema Nacional de Acreditación de la Educación Superior (SINAES). (2009). Manual de acreditación oficial de carreras de grado del Sistema de Acreditación de la Educación Superior. San José, Costa Rica: Autor. Recuperado de http://derecho.ucr.ac.cr/sites/all/documentos/ Acreditacion/Gestion de Calidad/Manual de Acreditaci\%C3\%B3n Sinaes.pdf

Sistema Nacional de Acreditación de la Educación Superior (SINAES). (2011). Guía para la autoevaluación de carreras - con fines de acreditación oficial. San José, Costa Rica: Autor. Recuperado de https://www.sinaes.ac.cr/documentos/Guia para la Autoevaluacion para carreras de grado.pdf

Sistema Nacional de Acreditación de la Educación Superior (SINAES). (2013a). Guía para autoevaluación de carreras por conglomerados. San José, Costa Rica: Autor. Recuperado de https://www.sinaes.ac.cr/documentos/Guia de autoevaluacion por conglomerado SINAES.pdf

Sistema Nacional de Acreditación de la Educación Superior (SINAES). (2013b). Referencia metodológica básica para acreditación por conglomerados. San José, Costa Rica: Autor. Recuperado de https://www.sinaes.ac.cr/documentos/Referencia metodologia conglomerados.pdf

Villarruel, M. (2010). Calidad en la educación superior: Un análisis reflexivo sobre la gestión de sus procesos en los centros educativos de América. Revista Iberoamericana sobre Calidad, Eficacia y Cambio en Educación, 8(5), 110-118. Recuperado de http://www.redalyc.org/ pdf/551/55119084008.pdf 
doi: http://dx.doi.org/10.15359/ree.23-3.13

URL: http://www.una.ac.cr/educare

CORREO: educare@una.cr

Apéndice $A$

Tablas de la estructura de cada encuesta

Tabla A1: Descripción por secciones y tipo de pregunta de la encuesta autoevaluación conglomerado estudiantes primer ingreso de ambos bachilleratos

\begin{tabular}{lccc}
\hline \multicolumn{1}{c}{ Secciones } & Total de preguntas & \multicolumn{2}{c}{ Tipo de preguntas } \\
\cline { 2 - 4 } & & Abierta & Cerrada \\
\hline Datos Generales & 4 & 0 & 4 \\
Vida Estudiantil & 2 & 0 & 2 \\
Correspondencia con el Contexto & 2 & 1 & 1 \\
Plan de Estudios & 7 & 0 & 7 \\
Personal Académico & 1 & 0 & 1 \\
Personal Administrativo & 1 & 0 & 1 \\
Infraestructura & 12 & 2 & 10 \\
Centro de Información y Recursos & 12 & 3 & 9 \\
Equipo y Materiales & 5 & 0 & 5 \\
Metodología Enseñanza-Aprendizaje & 3 & 0 & 3 \\
Vida Estudiantil & 17 & 2 & 15 \\
Desempeño Estudiantil & 9 & 4 & 5 \\
Comentarios Finales & 16 & 0 & 1 \\
\hline Total & 76 & 12 & 64 \\
\hline
\end{tabular}

Nota: Elaboración propia. 
Tabla A2: Descripción por secciones y tipo de pregunta de la encuesta autoevaluación conglomerado estudiantes regulares de ambos bachilleratos

\begin{tabular}{lccc} 
& Total de preguntas & \multicolumn{2}{c}{ Tipo de Preguntas } \\
\cline { 2 - 4 } & & Abierta & Cerrada \\
\hline Datos Generales & 5 & 0 & 5 \\
Componente Vida Estudiantil & 3 & 0 & 3 \\
Correspondencia con el Contexto & 2 & 1 & 1 \\
Plan de Estudios & 7 & 0 & 7 \\
Personal Académico & 1 & 0 & 1 \\
Personal Administrativo & 1 & 0 & 1 \\
Infraestructura & 12 & 2 & 10 \\
Centro de Información y Recursos & 12 & 3 & 9 \\
Equipo y Materiales & 5 & 0 & 5 \\
Metodología Enseñanza-Aprendizaje & 3 & 0 & 3 \\
Gestión de carrera & 18 & 2 & 16 \\
Desempeño Estudiantil & 5 & 1 & 4 \\
Comentarios Finales & 15 & 1 & 0 \\
\hline Total & & 10 & 65 \\
\hline
\end{tabular}

Nota: Elaboración propia. 
doi: http://dx.doi.org/10.15359/ree.23-3.13

URL: http://www.una.ac.cr/educare

CORREO: educare@una.cr

Tabla A3: Descripción por secciones y tipo de pregunta de la Encuesta autoevaluación conglomerado Personal docente de ambos bachilleratos

\begin{tabular}{lccc}
\hline \multicolumn{1}{c}{ Secciones } & Total de preguntas & \multicolumn{2}{c}{ Tipo de Preguntas } \\
\cline { 2 - 4 } & & Abierta & Cerrada \\
\hline Datos Generales & 6 & 0 & 6 \\
Personal Académico & 7 & 3 & 4 \\
Personal administrativo & 1 & 0 & 1 \\
Infraestructura & 14 & 3 & 11 \\
Centro de información y recursos & 12 & 3 & 9 \\
Equipo y materiales & 4 & 0 & 4 \\
Desarrollo docente & 6 & 3 & 3 \\
Metodología Enseñanza-Aprendizaje & 1 & 0 & 1 \\
Plan de estudios & 17 & 2 & 15 \\
Gestión de carrera & 3 & 1 & 2 \\
Componente de investigación & 9 & 3 & 6 \\
Componente de extensión & 2 & 0 & 2 \\
Componente estudiantil & 7 & 0 & 7 \\
Comentarios finales & 90 & 19 & 0 \\
\hline Total & & 73 \\
\hline
\end{tabular}

Nota: Elaboración propia. 
Tabla A4: Descripción por secciones y tipo de pregunta de la Encuesta autoevaluación conglomerados Personal administrativo CIEMHCAVI-DE

\begin{tabular}{lccc} 
& Total de preguntas & \multicolumn{2}{c}{ Tipo de Preguntas } \\
\cline { 3 - 4 } & & Abierta & Cerrada \\
\hline Datos Generales & 6 & 0 & 6 \\
Personal Administrativo & 6 & 0 & 6 \\
Infraestructura & 3 & 0 & 3 \\
Materiales y Equipo & 2 & 0 & 2 \\
Gestión de la Carrera & 2 & 1 & 1 \\
Vida Estudiantil & 7 & 0 & 7 \\
Comentarios Finales & 1 & 1 & 0 \\
\hline Total & 27 & 2 & 25 \\
\hline
\end{tabular}

Nota: Elaboración propia.

Tabla A5: Descripción por secciones y tipo de pregunta de las Encuestas autoevaluación conglomerado Estudiantado graduado BEEFDR y BPSF

\begin{tabular}{lccc}
\hline \multirow{2}{*}{ Secciones } & Total de preguntas & \multicolumn{2}{c|}{ Tipo de Preguntas } \\
\cline { 3 - 4 } & & Abierta & Cerrada \\
\hline Datos Generales & 6 & 0 & 6 \\
Situación laboral & 5 & 1 & 4 \\
Correspondencia con el Contexto & 7 & 2 & 5 \\
Componente graduados & 14 & 3 & 11 \\
Comentarios finales & 1 & 1 & 0 \\
\hline Total & 33 & 7 & 26 \\
\hline
\end{tabular}

Nota: Elaboración propia. 
doi: http://dx.doi.org/10.15359/ree.23-3.13

URL: http://www.una.ac.cr/educare

CORREO: educare@una.cr

Tabla A6: Descripción por secciones y tipo de pregunta de la Encuesta autoevaluación conglomerado Entes empleadores

\begin{tabular}{lccc}
\hline \multirow{2}{*}{ Secciones } & Total de preguntas & \multicolumn{2}{c}{ Tipo de Preguntas } \\
\cline { 3 - 4 } & & Abierta & Cerrada \\
\hline Datos Generales & 9 & 2 & 7 \\
Correspondencia con el contexto & 2 & 0 & 2 \\
Plan de estudios & 11 & 2 & 9 \\
Comentarios finales & 1 & 1 & 0 \\
\hline Total & 23 & 5 & 18 \\
\hline
\end{tabular}

Nota: Elaboración propia. 